\title{
NEUROPSYCHOLOGICAL EVALUATION OF THE HIV PATIENT
}

Yaakov Stern, PhD

Soon after the medical manifestations of AIDS were recognized, it became clear that cognitive changes and dementia were associated with the disease. The next important observation was that these cognitive changes are not simply secondary to medical disabilities but are actual manifestations of the disease. ${ }^{16}$ Thus, the Centers for Disease Control modified the definition of AIDS to include dementia as a defining illness. ${ }^{3}$ Today, it is recognized that just as the medical manifestations of HIV have a wide spectrum that ranges from an asymptomatic stage through milder medical symptoms to full-blown AIDS, cognitive changes have a spectrum that ranges from subtle early manifestations to full-blown dementia. Thus, neuropsychological evaluation can be important for diagnosing dementia or for evaluating a patient's subtle complaints of disorganization or memory problems. In this article, the neuropsychological techniques that can be used to evaluate these cognitive changes are reviewed.

\section{ISSUES IN NEUROPSYCHOLOGICAL EVALUATION}

The neuropsychological approach uses standardized tests, with known reliability and validity, to measure performance in a series of

This research was supported by center Grant P50-MH43520 from NIMH/NIDA.

From the Departments of Neurology and Psychiatry, College of Physicians and Surgeons,

Columbia University, and the Sergievsky Center, New York, New York 
cognitive domains. To the extent that the normative values for a particular test have been established, it is then possible to compare any individual's performance to that expected based on others with a similar background. By applying this technique to a full range of cognitive domains, a profile of a patient's strengths and weaknesses can be established.

The neuropsychological tests utilized in an assessment battery come from many different sources. Some were developed for academic purposes, such as intelligence tests, whereas others are derived from experimental cognitive psychology paradigms. Each test is intended to measure performance representing some specific cognitive construct. One implicit assumption is that defective performance on a test implies some underlying impairment in the function of the brain. Therefore, determining test areas that yield defective performance can give insight into the underlying cause of those deficits. Clearly, before attributing performance to brain dysfunction, the myriad other factors that affect performance must be considered

\section{Normative Values}

Typically, performance on various tests is judged with reference to normative values. In the best case, these norms are derived from populations that are comparable to the patient being evaluated in terms of age, education, socioeconomic background, and so on, and scores significantly below mean expected values are indicative of performance deficit. In other cases, performance can be evaluated with reference to assumptions about abilities that the average person is expected to have. For example, we expect that the average person is able to repeat simple sentences or has basic capacities for learning and remembering.

Appropriate norms are not always available for individuals with HIV. For example, there is not a large resource or normative data for intravenous drug users, who make up a major proportion of those suffering with HIV disease.9. 20 Similarly, there are not good norms for minorities or Spanish speakers. Often, it is important to acquire local norms for tests by gathering data on a population that is similar to that seen in clinical practice.

\section{Premorbid Function}

A parallel issue is the concept of premorbid intelligence. When evaluating change in cognition, it is typically in reference to a hypothetical baseline of performance ability. Therefore, for example, someone who is mentally retarded is not considered demented as poor performance on tests does not represent a decline from premorbid values. In better studied populations, it is often possible to estimate a premorbid intelligence from various aspects of demographics such as age, education, and gen- 
der. Formulas exist for calculating the Wechsler Adult Intelligence ScaleRevised intelligence quotient in this manner. In more nonstandard populations, however, this is not possible. One approach taken by the neuropsychologist is to utilize aspects of the neuropsychological evaluation that tap cognitive processes that typically are spared. For example, patients with mild and even moderate dementia perform well on tests that tap "old stores" of knowledge such as vocabulary. Performance on these tests then can be used as an indicator of previous abilities. Alternately, it can be assumed that tests eliciting the highest scores are most indicative of the premorbid level of ability, whether or not they are considered reflective of abilities that are not expected to change.

The new adult reading test (NART) ${ }^{15}$. is often used as an index of premorbid intelligence. This test requires the patient to read a series of words that are unusual in pronunciation. Performance can be translated into an index of education and premorbid intelligence. Another commonly used assessment of premorbid intelligence is the Vocabulary subtest of the WAIS-R. ${ }^{22}$

By far the best approach is to make use of records of previous performance on similar tests. When these are not available it may be necessary to follow a patient over time to determine whether performance is declining.

Again, this issue is most salient for understudied populations such as minorities, individuals with different cultural backgrounds, and those with low educational attainment. In all of these cases, it may be particularly difficult to determine whether a specific level of performance represents a decline from premorbid levels.

\section{Output of the Neuropsychological Evaluation}

The results of the neuropsychological evaluation are best considered in the same context as the results of other diagnostic testing that might be obtained by a physician. The minimum that a neuropsychological evaluation yields is an extensive investigation of the present abilities of the patient. In these cases, the studies may not lead to a definitive diagnosis, but they are useful for determining the patient's present capacities, tracking him or her in the future, and advising the patient and family.

In some cases, the evaluation can suggest that additional diagnostic tests are useful. For example, if a patient's pattern of performance deviates substantially from that typically expected at his or her stage of HIV disease, the possibility of other concomitant conditions is enhanced. Evaluation can suggest the utility of MR imaging, psychiatric consultation, or more intensive EEG recording.

Of course, many times the neuropsychologist can offer a tentative diagnosis or discuss the possible diagnoses compatible with the test findings.

The more information the examiner has at the start, the more directly the issues can be addressed. For example, if MR imaging has 
revealed the presence of a particular lesion, the examination can be tailored to evaluate its possible contribution to the patient's performance. In this context, the examination is not an exploration of tests' ability to detect a lesion but a contribution to understanding the implications of the lesion.

Similarly, the more explicit the referral question, the more likely the evaluation will yield useful information. A useful referral describes the differential diagnosis being entertained. Alternately, the physician or family simply want to document the patient's current condition or further explore some specific aspect of the patient's performance such as language functioning.

\section{Issues Specific to HIV Disease}

As neuropsychology developed, there was an attempt to map various aspects of cognitive functioning to specific cortical or subcortical areas or to networks of areas in the brain. Although this approach was particularly suitable for focal brain injuries such as bullet wounds or stroke, in degenerative disease such as Alzheimer's disease, neuropsychologists often must resort to describing observed deficits across different cognitive domains such as memory, language, or visuospatial function without reference to areas of focal brain damage. Still, even in these more diffuse illnesses, it is often possible to establish particular patterns of deficit that are typical of a specific condition and that are suggestive of preferential degeneration of specific brain areas.

The primary cognitive change associated with HIV disease is based on the direct effect of the virus on neuronal function. ${ }^{1}$ Although the mode of this effect still is researched intensively, it is recognized that the disease has a predilection for particular areas of the brain. Early pathologic studies of AIDS and AIDS dementia complex found that abnormalities were predominantly in the white matter and subcortical structures, sparing the cortex. ${ }^{13}$ Complicating the evaluation of the effects of HIV itself on cognitive function, however, is the fact that HIV is associated with both central and peripheral medical and neurologic conditions that also might affect cognitive function. Opportunistic infections, including toxoplasmosis and cryptococcosis; viral infections, such as progressive multifocal leukoencephalopathy and cytomegalovirus; and central nervous system lymphoma are some of the diseases that occur in the central nervous system and can affect a patient's cognition (see reference 18 for review). In addition, the effects of the various peripheral opportunistic infections and diseases associated with the AIDS syndrome can weaken a patient, cause delirium, affect their motivation, and otherwise compromise their day-to-day cognitive performance. Therefore, these conditions must be taken into account when interpreting the results of a cognitive evaluation.

Another aspect of the neuropsychological evaluation of HIV infection that requires consideration is the choice between using standard and 
more experimental assessments. The typical clinical neuropsychological battery in most hospital centers consists of a series of standard tests that have been proven useful for evaluating most conditions. These tests are not designed to optimally assess specific aspects of cognitive function in the manner of assessments used in cognitive psychology. Some subtle decrements in performance in HIV-positive individuals are best assessed with measures of speeded cognitive processing. These tasks typically are computerized tasks that emerge from the cognitive psychology laboratories. Unfortunately, they have not reached the point at which they are as standardized as many of the paper-and-pencil tasks that psychologists use. Therefore, there is a trade-off between breadth of application and ease of interpretation that is available from standard batteries and the ability to pinpoint specific or subtle deficits that might be obtained from more experimental tasks.

\section{NEUROPSYCHOLOGICAL ASSESSMENT OF HIV PATIENTS}

The cognitive areas assessed by the neuropsychological evaluation are relatively standard. The instruments used, however, are determined in part by the current capacities of the patient. Thus, a referral for subtle cognitive complaints in a relatively mild symptomatic patient would require a different set of evaluations than would a dementia work-up. The more general headings in Table 1 summarize the cognitive areas that are typically covered in a full neuropsychological evaluation.

The extent to which each of these areas is covered depends on the level of the patient and the degree of subtlety required of the evaluation. For example, in the more advanced patient, a simple assessment of memory, perhaps asking the patient to remember several words over a period of time or to remember a brief story might be sufficient. In a more intact patient, however, intensive evaluations of verbal and nonverbal shortterm and long-term memory would be required. The following discussion reviews some recommendations for selection of neuropsychological batteries in the early and more advanced patient with HIV infection.

\section{Early Disease}

After dementia was recognized in AIDS, the search began for the earliest point in HIV infection at which cognitive changes may occur. Some controversy still remains about whether individuals who are HIV positive but medically asymptomatic evidence cognitive changes. Although several large studies have found no performance differences between these individuals and matched controls, ${ }^{5,8,10,12}$ there are a sufficient number of published studies to suggest that subtle performance differences on neuropsychological tests exist. ${ }^{6,9,19,21}$ These differences have been noted in several areas including speeded cognitive tasks (on 
Table 1. NIMH NEUROPSYCHOLOGICAL BATTERY

A. Indication of Premorbid Intelligence

1. Vocabulary (WAIS-R)*

2. National Adult Reading Test (NART)

B. Attention

1. Digit Span (WMS-R)

2. Visual Span (WMS-R)*

C. Speed of Processing

1. Sternberg Search Task

2. Simple and Choice Reaction Time

3. Paced Serial Addition Test (PASAT)*

D. Memory

1. California Verbal Learning Test (CVLT)*

2. Working Memory Test

3. Modified Visual Reproduction Test

E. Abstraction

1. Category Test

2. Trail Making Test, Parts A and B

$F$. Language

1. Boston Naming Test

2. Letter and Category Fluency Test

G. Visuospatial

1. Embedded Figures Test

2. Money's Standardized Road-Map Test of Direction Sense

3. Digit Symbol Substitution

H. Construction Abilities

1. Block Design Test

2. Tactual Performance Test

I. Motor Abilities

1. Grooved Pegboard

2. Finger Tapping Test

3. Grip Strength

J. Psychiatric Assessment

1. Diagnostic Interview Schedule (DIS)

2. Hamilton Depression Scale*

3. State-Trait Anxiety Scale*

4. Mini-Mental State Examination

"Indicates test in the abbreviated version of the battery.

more experimental tasks), memory, and certain aspects of language. Whether these subtle changes have any clinically significant effect on a patient's day-to-day behavior remains to be seen. In addition, it still has not been established whether these early, subtle changes are the harbingers of more severe changes to occur later. Be that as it may, it is recognized by most groups that once a patient becomes more medically symptomatic, but still without an AIDS defining illness, neuropsychological changes are more likely to be seen. In an attempt to differentiate these early cognitive changes from dementia, the American Academy of Neurology task group 7 defined a disorder known as minor motor cognitive disorder that is meant to encompass early cognitive changes that have some subtle implications for day-to-day function. The criteria for this disorder are presented in Table 2.

If a clinician is to attempt to establish this diagnosis using neuropsy- 
Table 2. DIAGNOSTIC CRITERIA FOR HIV-1-ASSOCIATED COGNITIVE/MOTOR DISORDER

1. Cognitive/motor/behavioral abnormalities (both $A$ and $B$ )

A. At least two of the following:

1. Impaired attention or concentration

2. Mental slowing

3. Impaired memory

4. Slowed movements

5. Incoordination

6. Personality change.

B. Acquired cognitive/motor abnormality verified by clinical neurologic examination or neuropsychological testing (e.g., fine motor speed, manual dexterity, perceptual motor skills, attention/concentration, speed of processing information, abstraction/reasoning, visuospatial skills, memory/learning, or speech/language).

2. Disturbances from No. 1 cause mild impairment of work or activities of daily living.

3. Does not meet criteria for HIV-1-Associated Dementia Complex or myelopathy.

4. No evidence of active CNS opportunistic infection, malignancy, or severe systemic illness. No. 1 not attributable solely to substance abuse or other psychiatric disorders.

chological tests, the specific categories of cognitive change mentioned in the definition must be assessed. A list of specific areas of function, however, does not truly encompass the typical cognitive complaints of the HIV-positive patient. Often, patients complain of an inability to organize their activities well. For example, a patient might report difficulty paying bills because he or she has difficulty coordinating all the activities required. More subtle complaints are often in terms of less efficiency in carrying out tasks at work, even though the job eventually gets done. These subtle complaints are often difficult to document in a neuropsychological examination because most tasks do not address these issues. Sometimes tests of executive function that assess the ability to plan, sequence, and monitor ongoing behavior can pick up some of these subtle complaints. In addition, tests of attention or of speeded cognitive function may pinpoint reductions in performance that parallel clinical complaints.

The most extensive published recommended neuropsychological battery to assess early cognitive changes in HIV disease was developed at a National Institute of Mental Health Workshop on neuropsychological assessment approaches in HIV. ${ }^{2}$ Although this battery was designed primarily for research purposes, it, or its short version, can serve as a model for constructing a clinical evaluation as well. The domains covered and specific tests used are listed in Table 1 . The complete battery takes up to 9 hours, and was intended to encompass a range of cognitive function that might be affected by HIV disease. For a full description of each test, the reader is referred to the original presentation of the battery. The battery includes several "experimental tests" that, as discussed previously, have not been truly standardized across neuropsychology laboratories and do not have a well-established normative base. Still, these tasks might be used at a particular site if it is anticipated that many patients will be evaluated and local norms could be developed. It should 
be emphasized that, for every test chosen by the work group to assess a particular cognitive domain, there are several others that would be equally suitable. It is only in the choice of the more experimental tests, the Sternberg test and simple and choice reaction time, that decisions were guided by actual findings in the field suggesting the utility of these particular tests for detecting early cognitive change in patients with HIV disease.

A shorter battery that has been used by the Multicenter AIDS Collaborative Studies (MACS) groups for the evaluation of a large number of HIV-positive individuals ${ }^{17}$ is useful, mainly because of the great amount of normative data available. Although it is insufficient for assessing very early subtle changes, it can serve as the nucleus for evaluating more advanced changes in later stages of the disease. The primary value of this battery, however, is that extensive normative data have been acquired on a large number of HiV-positive individuals. The components of this battery are (1) Symbol Digit Modalities, (2) WAIS-R Digit Span, (3) Rey Auditory Verbal Learning Test, (4) Controlled Oral Word Association, and (5) Grooved Pegboard. As can be seen from this list, this battery is essentially a subset of the NIMH battery, with the exception that the Rey is used here instead of the California Verbal Learning test.

Although not typically included in the published batteries specifically devoted to HIV, the Wechsler Adult Intelligence Scale-Revised $(\text { WAIS-R })^{20}$ is a standard component of most clinical batteries intended to explore subtle cognitive complaints. The advantages of this test are its standard usage across many settings (yielding comparability), the good normative base available for it, and the wealth of information regarding a wide range of cognitive functioning that it yields. Because the WAIS-R is a standard tool, most neuropsychologists are adept in interpreting results of this battery and in evaluating the relative significance of various patterns of performance.

\section{Dementia}

The American Academy of Neurology Work Group also has recommended diagnostic criteria for dementia in HIV (Table 3 ). ${ }^{7}$ Again, these

\section{Table 3. DIAGNOSTIC CRITERIA FOR HIV-1-ASSOCIATED DEMENTIA COMPLEX}

1. Acquired abnormality in at least two of the following cognitive abilities: attention/concentration, speed of processing information, abstraction/reasoning, visuospatial skills, memory/learning, and speech/language.

2. Cognitive impairment causes impairment in work or activities of daily living.

3. One of the following:

A. Acquired abnormality in motor functioning verified by clinical examination and/or neuropsychological tests

B. Decline in motivation or emotional control.

4. Cognitive deficit demonstrable in the absence of clouding of consciousness.

5. If another potential cause is present (e.g., depression, substance abuse, and so on), it is not the cause of the cognitive, motor, or behavioral symptoms or signs. 
definitions list a series of specific cognitive areas that, by definition, may be involved in the dementia complex. In addition, motor and affective deficits are included. Therefore, a clinical, neuropsychological evaluation of a dementing disorder would be well advised to assess these areas. Although it is well established that dementia occurs in HIV patients, ${ }^{1,14,16}$ the definition is still somewhat in flux and, therefore, it might be advisable to extend the evaluation of a patient referred for a possible HIVrelated dementia to include the more extensive evaluation that might be given to individuals referred for any dementia evaluation such as Alzheimer's disease. As mentioned previously, the WAIS-R, in combination with a range of tests from the categories described in Table 1, would yield a comprehensive battery.

Another clinical issue is that of following patients with more advanced illness and more clear-cut cognitive decline. In this case, the intent is no longer diagnosis but clinical care and follow-up. For these purposes, a brief battery or even a mental status evaluation such as the Folstein Mini-Mental State Examination might be sufficient. Although tests such as these are relatively crude, regular administration can yield some information about improvement or decline in function through time.

\section{SUMMARY}

The design of a neuropsychological evaluation of the HIV patient must follow the standard procedures used in any clinical condition. A wide range of cognitive functions should be evaluated to develop a pattern of strengths and weaknesses. Specific attention should be devoted to areas that have been reported to be affected in HIV. However, because concomitant opportunistic infections of the central nervous system are common, evaluation cannot be limited to only these areas. Finally, the special challenge of evaluating people for whom the normative base is limited, including groups such as minorities and drug users, must be kept in mind.

\section{References}

1. Brew BJ, Rosenblum M, Price RW: AIDS dementia complex and primary HIV brain infection. J Neuroimmunol 10:1330-1340, 1988

2. Butters N, Grant I, Haxby J, et al: Assessment of AIDS-related cognitive changes: Recommendations of the NIMH Workgroup on Neuropsychological Assessment Approaches. J Clin Exp Neuropsychol 12:963-978, 1990

3. Centers for Disease Control: Classification system for human T-lymphotropic virus type III/lymphadenopathy-associated virus infections. MMWR 35:334, 1986

4. Folstein MF, Folstein SE, McHugh P: "Mini-Mental State." A practical method for grading the cognitive state of patients for the clinician. J Psychiatr Res 12:189-198, 1975

5. Goethe K, Mitchell J, Marshall W, et al: Neuropsychological and neurological function of human immunodeficiency virus seropositive asymptomatic individuals. Arch Neurol 46:129-133, 1989

6. Grant I, Atkinson J, Hesselink J, et al: Evidence for early central nervous system 
involvement in the acquired immunodeficiency syndrome and other HIV infections. Ann Intern Med 107:828-836, 1987

7. Janssen RS, Cornblath DR, Epstein LG, et al: American Academy of Neurology AIDS Task Force. Nomenclature and research case definitions for neurologic manifestations of human immunodeficiency virus-type 1 (HIV-1) infection. Neurology 41:778-785, 1991

8. Janssen RS, Saykin AJ, Cannon L, et al: Neurological and neuropsychological manifestations of HIV-1 infection: Association with AIDS-related complex but not asymptomatic HIV-1 infection. Ann Neurol 26:592-600, 1989

9. Marder K, Stern Y, Malouf R, et al: Neurological and neuropsychological manifestations of HIV infection in intravenous drug users without AIDS: Relationship to head injury. Arch Neurol 49:1169-1177, 1992

10. McArthur J, Cohen B, Selnes O, et al: Low prevalence of neurological and neuropsychological abnormalities in otherwise healthy HIV-1 infected individuals: Results from the multicenter AIDS cohort study. Ann Neurol 26:601-609, 1989

11. McKegney FP, O'Dowd MA, Feiner C, et al: A prospective comparison of neuropsychologic function in HIV-seropositive and seronegative methadone-maintained patients. AIDS 4:565-569, 1990

12. Miller EN, Selnes OA, McArthur JA, et al: Neuropsychological performance in HIV-1 infected homosexual men: The Multicenter AIDS Cohort Study (MACS). Neurology

13. Navia B, Cho E-S, Petito C, et al: The AIDS dementia complex: II. Neuropathology. Ann Neurol 19:525-535, 1986

14. Navia BA, Jordan BD, Price RW: The AIDS dementia complex: I Clinical features. Ann Neurol 19:517-524, 1986

15. Nelson HE, O'Connell A: Dementia: The estimation of premorbid intelligence levels using the new adult reading test. Cortex 14:234-244, 1978

16. Price RW, Brew B, Sidtis J, et al: The brain in AIDS: Central nervous system HIV-1 infection and AIDS dementia complex. Science 239:586-592, 1988

17. Selnes OA, Jacobson L, Machado AM, et al: Normative data for a brief neuropsychological screening battery: Multicenter AIDS Cohort Study. Percept Mot Skills 73:539-550, 1991

18. Stern $Y$, Marder K: The neurology and neuropsychology of HIV infection. In Boller F, Grafman J (eds): The Handbook of Neuropsychology, vol 5. Amsterdam, Elsevier, 1991, pp 265-284

19. Stern $Y$, Marder K, Bell $K$, et al: Multidisciplinary baseline assessment of gay men with and without HIV infection: III. Neurological and neuropsychological findings. Arch Gen Psychiatry 48:131-138, 1991

20. Wechsler D: Wechsler Adult Intelligence Scale-Revised. New York, The Psychological Corporation, 1981

21. Wilke FL, Eisdorfer CE, Morgan R, et al: Cognition in early human immunodeficiency virus infection. Arch Neurol 47:433-440, 1990

Address reprint requests to

Yaakov Stern, PhD

Neurological Institute

710 West 168th Street

New York, NY 10032 